\title{
Recalcitrant pemphigus foliaceous and immunosuppressive treatment complications
}

\author{
Simon Tso*, Rafaella Siang, Sue A Chan, Nazipha F Mahmood and Weronika Szczecinska \\ Department of Dermatology, University Hospitals Coventry and Warwickshire NHS Trust, Coventry, CV2 2DX, UK
}

\section{Brief history}

A 79 year-old Caucasian man presented with a 3-month history of a tender and mildly pruritic erythematous eruption on his head, neck and upper back. There was no preceding illness and the patient has never smoked. Over the next one month, the rash progressed to the rest of his body despite the use of potent (betamethasone valerate $0.1 \%$ and fluocinonide $0.05 \%$ ) and very potent (clobetasol propionate $0.05 \%$ ) topical corticosteroids, oral prednisolone at $60 \mathrm{mg}$ once daily and 3 consecutive days of intravenous methylprednisolone at 1gram/ day. The rash was associated with rapid and unintentional weight loss of over $15 \mathrm{~kg}$ despite a good appetite and nutritional supplements in a 3-month period, which could potentially be explained by augmented trans-epidermal water and electrolyte loss, thermal dysregulation and increased skin blood flow [1]. At the height of his skin disease, clinical examination revealed a single intact blister on his left flank, a confluent erythematous rash with scattered areas of superficial erosion on his face and torso (Figure 1), widespread erythematous patches with superficial erosions on his limbs with no mucosal involvement. Investigations revealed a neutrophilia $17.41 \times 10^{\%} / \mathrm{L}$ and normal C-Reactive Protein. Computerized tomography scan of chest, abdomen and pelvis revealed a right middle lobe pulmonary nodule that was later biopsied. PCR revealed aspergillus species, which resolved radiologically two months later following further imaging. A skin biopsy showed acanthosis and inflammatory changes with eosinophils. Direct immunofluorescence

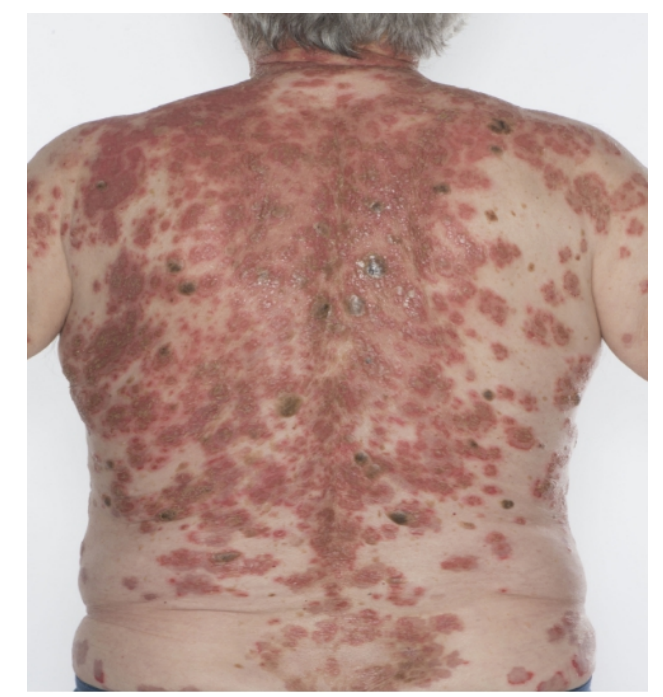

Figure 1. Shows a confluent erythematous exfoliative rash on the patient's back with a background of multiple inflamed seborrhoeic keratosis. Photograph taken at the time of initial histological diagnosis of pemphigus foliaceous
(DIF) microscopy showed intercellular deposition of IgG in the epidermis with $\mathrm{C} 3$ at the basement membrane zone. ELISA demonstrated antibodies against DSG1 at $190 \mathrm{U} / \mathrm{ml}$ (no antibodies against DSG3) which confirmed the diagnosis of pemphigus foliaceous [2]. His skin disease partially responded to the combination treatment of oral prednisolone at $40 \mathrm{mg} /$ day and mycophenolate mofetil at $2 \mathrm{~g} /$ day but continued to develop intermittent new eroded plaques on his limbs whenever his oral prednisolone was reduced. Despite receiving two doses of lgram Rituximab infusion two weeks apart for recalcitrant pemphigus foliaceous [3], he continued to develop new annular rash on his torso and limbs. During the treatment period, his pemphigus foliaceous was complicated by recurrent herpes simplex virus type 1 skin infections requiring repeated courses of valganciclovir, persistent lymphopenia (due to mycophenolate mofetil), Cushingoid physique and steroid-induced diabetes mellitus requiring insulin treatment. Unbeknown to the clinicians, the patient developed tinea incognito at some point in time in the patient journey due to immunosuppression that was uncovered when the patient was reviewed at a tertiary immunobullous disease service. The skin rash fully resolved following a 4-week course of oral terbinafine at $250 \mathrm{mg}$ once daily and Canestan HC (clotrimazole and hydrocortisone acetate) cream once daily. His immunosuppressive treatments were stopped leading to full resolution of his steroid-induced diabetes mellitus and his pemphigus foliaceous continued to be in remission 1 month after.

\section{Discussion}

This patient experienced clinically significant weight loss, which could have benefited from early and intensive nutritional support, steroid-induced diabetes mellitus and infections (bacterial, viral and fungal) are well recognised complications of immunosuppression and tinea incognito and herpetic infections should be considered as differential diagnosis in patients with clinically recalcitrant pemphigus foliaceous.

\section{Conclusion}

This case provided a useful overview about the sequelae of pemphigus foliaceous and possible complications from its treatments.

${ }^{*}$ Correspondence to: Simon Tso, Department of Dermatology, University Hospitals Coventry and Warwickshire NHS Trust, Coventry, CV2 2DX, UK, Tel: (079) 26578452; E-mail: simontso@doctors.org.uk

Key words: vasectomy, sexual reproduction, awareness, family planning

Received: November 16, 2018; Accepted: December 10, 2018; Published: December 14, 2018 


\section{References}

1. Mohd Noor N, Hussein SH (2103) Transepidermal water loss in erythrodermic patients of various aetiologies. Skin Res Technol 19: 320-323. [Crossref]
2. Marco CD (2106) Pemphigus: Pathogenesis to Treatment. R I Med J (2013) 99: 28-31. [Crossref]

3. Harman KE, Brown D, Exton LS, Groves RW, Hampton PJ, et al. (2017) British Association of Dermatologists' guidelines for the management of pemphigus vulgaris 2017. Br J Dermatol 177: 1170-1201. [Crossref]

Copyright: (C2018 Tso S. This is an open-access article distributed under the terms of the Creative Commons Attribution License, which permits unrestricted use, distribution, and reproduction in any medium, provided the original author and source are credited. 